\title{
Transvaginal natural orifice transluminal endoscopic surgery for uterosacral ligament suspension: pilot study of 35 cases of severe pelvic organ prolapse
}

\author{
Zhiying Lư ${ }^{\dagger}$, Yisong Chen ${ }^{\dagger}$, Xiaojuan Wang, Junwei Li, Keqin Hua ${ }^{*}$ and Changdong Hu ${ }^{*}$ (I)
}

\begin{abstract}
Background: To describe the short-term outcomes of transvaginal natural orifice transluminal endoscopic surgery (vNOTES) for uterosacral ligament suspension (USLS) in patients with severe prolapse.

Methods: This was a retrospective study of patients with severe prolapse ( $\geq$ stage 3 ) who underwent vNOTES for USLS between May 2019 and July 2020. The Pelvic Organ Prolapse Quantification (POP-Q) score, Pelvic Organ Prolapse/Urinary Incontinence Sexual Questionnaire short form (PISQ-12) and Pelvic Floor Inventory-20 (PFDI-20) were used to evaluate physical prolapse and quality of life before and after VNOTES for USLS.

Results: A total of 35 patients were included. The mean operative duration was $111.7 \pm 39.4 \mathrm{~min}$. The mean blood loss was $67.9 \pm 35.8 \mathrm{ml}$. Statistically significant differences were observed between before and after VNOTES USLS in $\mathrm{Aa}(+0.6 \pm 1.7$ versus $-2.9 \pm 0.2), \mathrm{Ba}(+1.9 \pm 2.2$ versus $-2.9 \pm 0.3), \mathrm{C}(+1.5 \pm 2.2$ versus $-6.9 \pm 0.9), \mathrm{Ap}(-1.4 \pm 1.0$ versus $-3.0 \pm 0.1)$ and $\mathrm{Bp}(-1.1 \pm 1.4$ versus $-2.9 \pm 0.1)(\mathrm{P}<0.05$ for all). The mean pre- and postoperative PFDI-20 score was $19.9 \pm 6.7$ and $3.2 \pm 5.4$, respectively, and the mean pre- and postoperative PISQ-12 score was $24.8 \pm 2.3$ and $38.3 \pm 4.1$, respectively ( $P<0.05$ for both). During 1-13 months of follow-up, there were no cases of severe complications or recurrence.

Conclusions: VNOTES for USLS may be a feasible technique to manage severe prolapse, with promising short-term efficacy and safety. Larger studies with more patients and longer follow-up periods should be performed to evaluate the long-term efficacy and safety profile of vNOTES for USLS.
\end{abstract}

Keywords: Transvaginal natural orifice transluminal endoscopic surgery, Pelvic organ prolapse, Uterosacral ligament suspension

\section{Background}

Pelvic organ prolapse (POP) is a common benign condition with a high risk of occurrence of $40-60 \%$ in elderly women [1] and a reported lifetime risk of surgical

*Correspondence: huakeqinjiaoshou@163.com; 13651712669@163.com †Zhiying Lu and Yisong Chen are co-first authors and contributed equally to this work

Department of Gynecology, The Obstetrics and Gynecology Hospital

of Fudan University, 128 Shenyang RD, Shanghai 200090, China intervention of $10-20 \%$ [2]. The gold-standard surgical treatment for POP is resuspension of the pelvic anatomy by native tissue repair or mesh repair. Mesh repair is associated with high rates of surgical complications and postoperative adverse events [3]. On 16 April 2019, transvaginal repair products were withdrawn from the market by the FDA [4]. Native tissue repair has received increasing attention in reconstructive pelvic surgery. Uterosacral 
ligament suspension (USLS) is a commonly performed procedure to support the vaginal apex [5].

Many procedures, such as laparoscopic procedures via an abdominal approach and transvaginal procedures, have been described for USLS. However, transvaginal USLS carries up to an $11 \%$ risk of ureteral injury, which is attributed to poor visibility [6, 7]. Unger et al. performed the largest retrospective series examining transvaginal USLS and found a $4.5 \%$ risk of intraoperative ureteral kinking [8]. The abdominal approach via laparoscopy offers improved visibility and allows these vaginal complications to be prevented, resulting in better suspension than the vaginal approach $[9,10]$. Houlihan et al. compared uterosacral vault suspension at the time of hysterectomy via a laparoscopic versus vaginal approach. The results showed less ureteral kinking ( 0 vs. $14 \%, p=0.023)$, urinary retention $(15 \%$ vs. $31 \%, \mathrm{p}=0.024)$, and symptomatic recurrence ( $24 \%$ vs. $41 \%, \mathrm{p}=0.046)$ in the laparoscopic group [11].

In recent years, some scholars have reported the advantages of transvaginal natural orifice transluminal endoscopic surgery (vNOTES) for USLS, which include no incisional pain and a better cosmetic outcome than laparoscopy via the abdominal approach, as well as direct visualization of key structures, such as the ureters and rectum, which is not available with the traditional transvaginal approach $[12,13]$. At present, there have been few studies on this topic. Therefore, it is clinically important to thoroughly research the safety and efficacy of this procedure given the paucity of existing data.

In this study, we performed USLS for POP using the vNOTES approach. Our objective was to describe the short-term outcomes of vNOTES for USLS in patients with severe apical prolapse.

\section{Methods}

\section{Patients}

We retrospectively collected data on all cases of vNOTES for USLS at the Obstetrics and Gynecology Hospital of Fudan University between May 2019 and July 2020. The inclusion criteria were as follows: (1) age 25-79 years; (2) severe apical prolapse ( $\geq$ stage 3 ); (3) desire for preservation of coital function; (4) first surgical treatment for POP; and (5) refusal of mesh implantation. The exclusion criteria were as follows: (1) inability to tolerate surgery; (2) coagulation dysfunction; (3) severe vaginal ulcers; (4) history of severe adhesions, a fixed uterus or strong pelvic adhesions noted on pelvic examination; (5) inability to tolerate the Trendelenburg position; and (6) suspicion of gynaecological malignancy. This retrospective study was approved by our institutional review board before any data were collected (no. 2019-32).
We extracted the following information from medical records: patient demographics (age, body mass index, parity, and history of prior hysterectomy or prolapse repair) and perioperative outcomes. Perioperative data included the operative duration, blood loss, intraoperative complications (transfusion or injury), postoperative complications (infection, urinary retention, persistent pain, haematoma, constipation, dyspareunia, de novo stress urinary incontinence or deep vein thrombosis), length of postoperative hospital stay, and hospitalization costs. We also assessed the change in physical prolapse with Pelvic Organ Prolapse Quantification (POP-Q). Two validated questionnaires, the Pelvic Organ Prolapse/ Urinary Incontinence Sexual Questionnaire short form (PISQ-12) and Pelvic Floor Inventory-20 (PFDI-20), were completed before and at least 3 months after vNOTES for USLS to assess the impact on quality of life. Postoperative follow-up visits were scheduled at 1 month, 3 months, 6 months, 1 year and 2 years after surgery. Any POP-Q score greater than or equal to $-1 \mathrm{~cm}$ was defined as recurrence [14].

\section{Surgical procedures}

All participants underwent surgery with general anaesthesia and standard operative care. Patients treated with hysterectomy received cefuroxime preoperatively. A single-port device with four trocars (HTKD Medical, China) and nonabsorbable or delayed absorbable sutures $(2-0$, Ethicon LLC, America) were used.

\section{Transvaginal hysterectomy with vNOTES for USLS}

This surgical procedure was originally described by Lowenstein et al. in 2019 [12]. In this study, the key steps were as follows (Fig. 1): Step 1: A routine transvaginal hysterectomy was performed (Fig. 1i). Step 2: The lap protector was placed from the vaginal orifice to the pelvic cavity through the vaginal vault, and then, the single-port platform was established (Fig. 1ii). Step 3: The ureters and uterosacral ligaments (USLs) were identified under single-port laparoscopy. If the ureter was found to be very close to the ipsilateral uterosacral ligament, a peritoneal release incision was performed to avoid kinking of the ureter. Step 4: One nonabsorbable suture was placed around the intermediate portion of the USL at the level of the ischial spine bilaterally, for a total of 4 stitches (Fig. 1iii). The sutures were then tugged slightly to confirm correct placement (Fig. 1iv). Step 5: The pelvic cavity was washed with normal saline, the single-port platform was removed, and the peritoneum was closed. Step 6: The above nonabsorbable sutures were attached to the ipsilateral cardinal ligament stump (Fig. 1v) and the pubocervical fascia of the anterior vaginal wall (Fig. 1vi). Step 7: Then, the above nonabsorbable sutures were attached to 


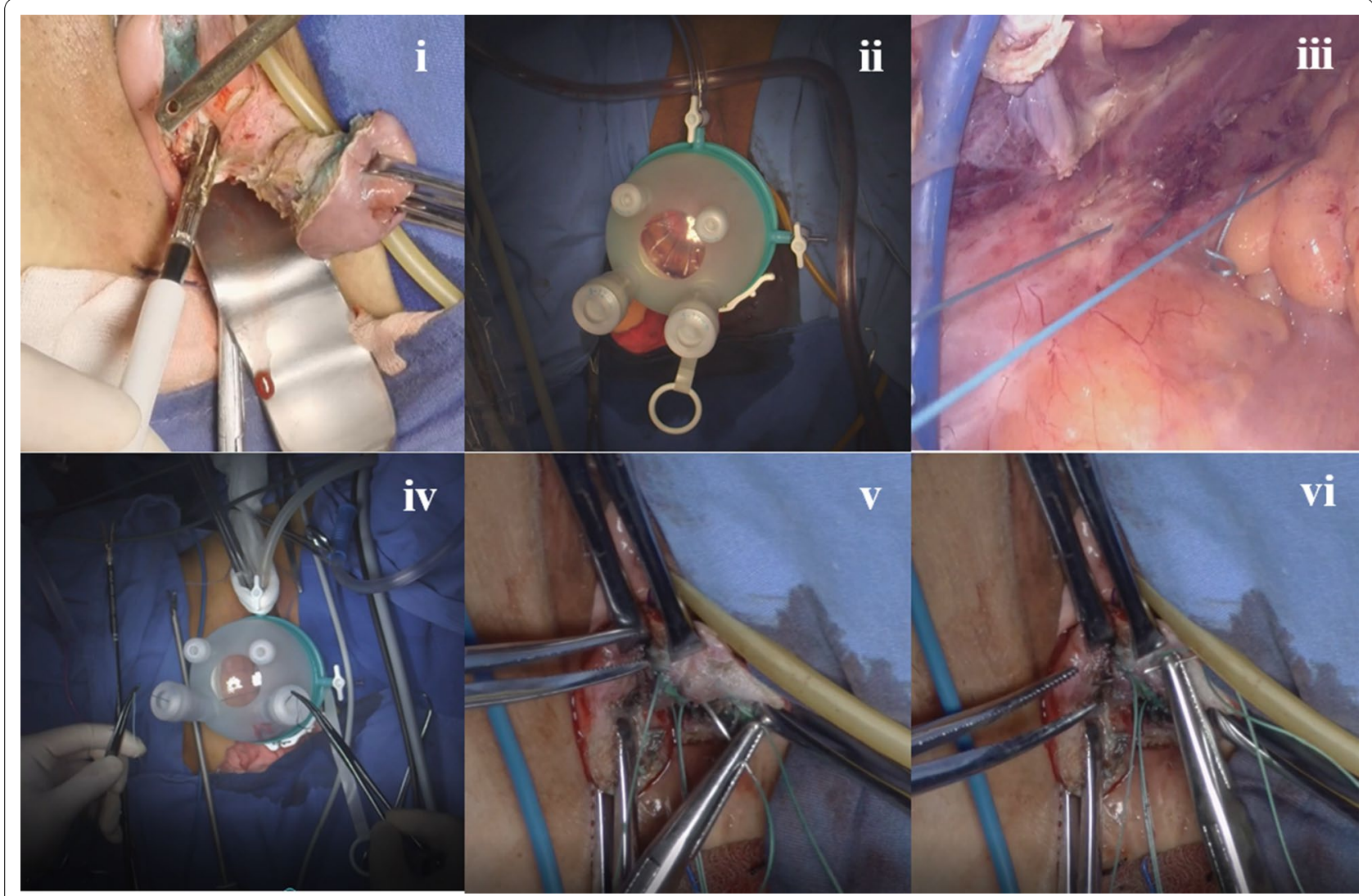

Fig. 1 Steps of transvaginal hysterectomy with transvaginal natural orifice transluminal endoscopic surgery (vNOTES) for uterosacral ligament suspension (USLS). i: Transvaginal hysterectomy. ii: Installation of the single-port platform. iii: Placement of one nonabsorbable suture around the intermediate portion of the USL at the level of the ischial spine bilaterally, for a total of 4 stitches (right USL shown). iv: Slight tugging of the sutures to confirm correct placement. v: Attachment of the above nonabsorbable sutures to the ipsilateral cardinal ligament stump (white arrow). vi: Attachment of the above nonabsorbable sutures to the pubocervical fascia (white arrow) of the anterior vaginal wall

the vaginal cuff and tied, suspending the vaginal cuff by the ligaments.

\section{Fertility preservation with vNOTES for USLS}

The single-port platform was placed from the vaginal orifice to the pelvic cavity through the posterior fornix. In these cases, apical support was achieved by suturing the USLs, shortening the ligaments (as mentioned above) (Fig. 2i) and reinforcing their attachment to the cervix (Fig. 2ii).

\section{Uterine preservation with vNOTES for USLS}

In patients who desire preservation of the uterus without a requirement for fertility, part of the cervix was excised according to the degree of cervical extension (Fig. 2iii), and finally, the residual cervix was reconstructed (Fig. 2iv). The other procedures were the same as those in fertility-preserving USLS.

Anterior or posterior colporrhaphy is necessary when the anterior or posterior compartment reaches stage
II prolapse. Perineal body repair was performed at the discretion of the operating surgeon such that the width of the vaginal orifice was more than that of 3 fingers at the end of the procedure. Anti-incontinence surgery was allowed according to the preoperative presence of stress urinary incontinence (SUI). The placement of a tensionfree midurethral sling (MUS) was performed in patients who were willing to undergo mesh placement. If not, urethral folding was performed. Gauze was inserted into the vagina for compression.

After surgery, routine postoperative care was provided. Cefuroxime was administered once postoperatively in patients treated with hysterectomy. All patients were then followed clinically; the longest follow-up duration was 13 months.

\section{Statistical analysis}

Data collection and statistical analyses were performed using IBM SPSS Statistics 24.0 software (IBM Corp., Armonk, New York, USA). All variables are presented 


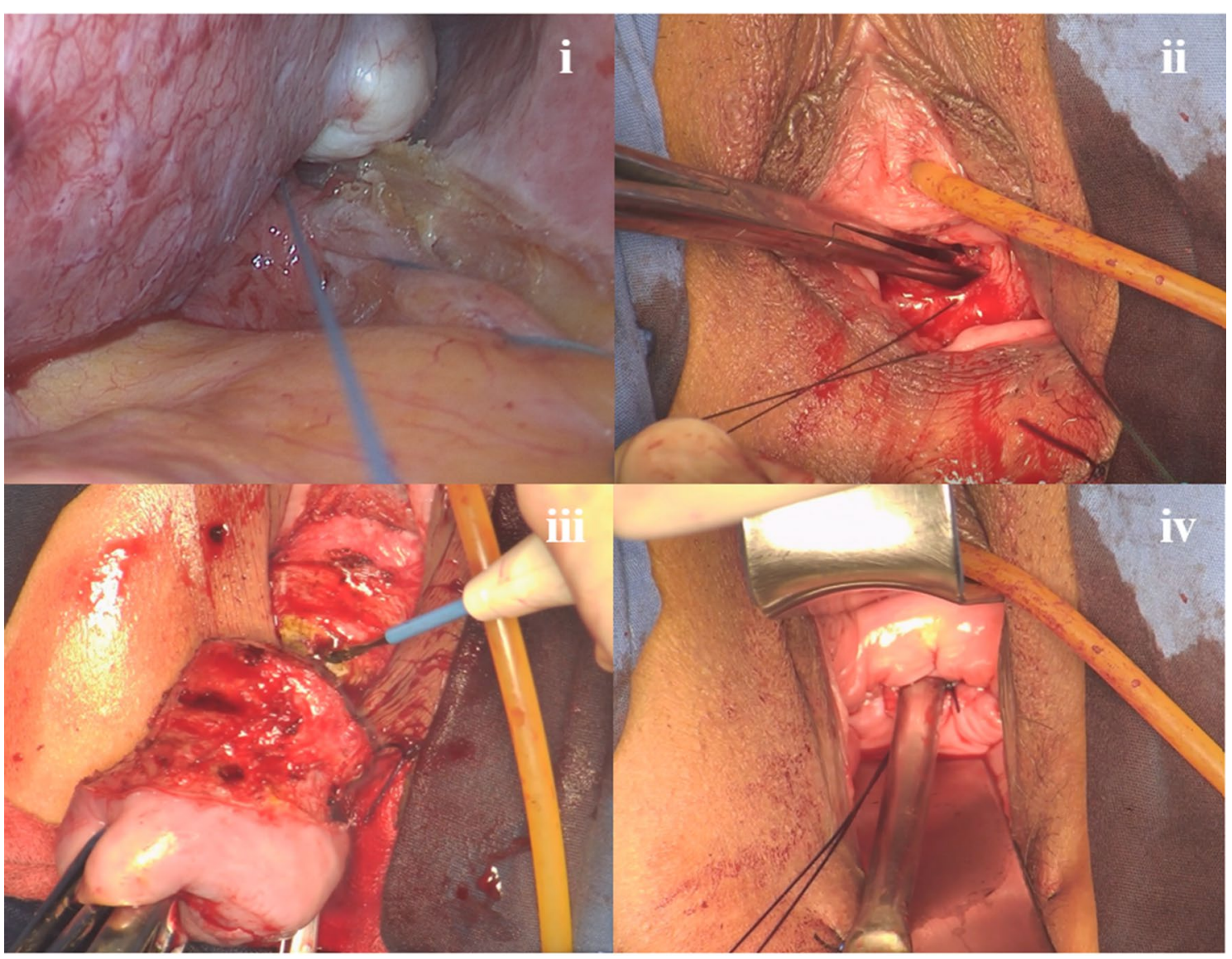

Fig. 2 Key steps of fertility-preserving or uterine-preserving vNOTES for USLS. i: Placement of one nonabsorbable suture around the intermediate portion of the USL at the level of the ischial spine bilaterally, for a total of 4 stitches (left USL shown). ii: Attachment of the above nonabsorbable sutures to the cervix. iii: Partial excision of the cervix. iv: Reconstruction of the residual cervix

as the mean and standard deviation (SD) or $\mathrm{n}$ and percentage (\%). Continuous variables were compared by Student's $t$ test. A P value $<0.05$ was considered statistically significant.

\section{Results}

In total, 35 patients underwent vNOTES for USLS; in one patient, vNOTES sacrocolpopexy was planned, but the patient was converted to vNOTES for USLS because of a suspected malignant lesion on the surface of the colon. The patient characteristics are shown in Table 1. One patient had previous undergone hysterectomy for POP without any suspension. Thirty-three patients (94.3\%) had stage III prolapse, and the other two patients (5.7\%) had stage IV prolapse.

Table 2 shows the perioperative outcomes and complications. Twenty patients (57.1\%) underwent hysterectomy, 2 patients $(5.7 \%)$ required fertility preservation, and 12 patients (34.3\%) required uterine preservation. Three patients underwent concomitant
Table 1 Patient characteristics

\begin{tabular}{ll}
\hline Variable & Values \\
\hline Number & 35 \\
Age (years) & $53.7 \pm 11.4$ \\
BMI $\left(\mathrm{kg} / \mathrm{m}^{2}\right)$ & $24.0 \pm 3.3$ \\
Parity & $1.6 \pm 0.8$ \\
Diabetes mellitus & $1(2.9)$ \\
Smoker & 0 \\
Prior hysterectomy & $1(2.9)$ \\
Prior prolapse repair & $1(2.9)$ \\
Prolapse stage & \\
Anterior & $2.5 \pm 0.7$ \\
Apical & $2.6 \pm 0.7$ \\
Posterior & $1.4 \pm 0.6$ \\
Baseline POP-Q stage & \\
III & $33(94.3)$ \\
IV & $2(5.7)$ \\
\hline
\end{tabular}

BMI body mass index, POP-Q Pelvic Organ Prolapse Quantification Values are shown as the mean \pm standard deviation or $\mathrm{n}(\%)$ 
Table 2 Perioperative and short-term outcomes $(n=35)$

\begin{tabular}{|c|c|}
\hline Variable & Values \\
\hline Intraoperative hysterectomy & $20(57.1)$ \\
\hline Preservation of fertility & $2(5.7)$ \\
\hline Preservation of uterus & $12(34.3)$ \\
\hline \multicolumn{2}{|l|}{ Concurrent surgery } \\
\hline Anti-incontinence surgery & $5(14.3)$ \\
\hline MUS surgery & $3(8.6)$ \\
\hline Urethral folding & $2(5.7)$ \\
\hline Anterior colporrhaphy & $24(68.6)$ \\
\hline Posterior colporrhaphy & $24(68.6)$ \\
\hline Perineal body repair & $24(68.6)$ \\
\hline Ovarian cystectomy & $2(5.7)$ \\
\hline Salpingo-oophorectomy & $10(28.6)$ \\
\hline Salpingectomy & $2(5.7)$ \\
\hline $\begin{array}{l}\text { Resection of a suspected malignant lesion on the } \\
\text { surface of the colon }\end{array}$ & $1(2.9)$ \\
\hline Operative duration (min) & $111.7 \pm 39.4$ \\
\hline Blood loss (ml) & $67.9 \pm 35.8$ \\
\hline Postoperative hospital stay (days) & $3.7 \pm 1.1$ \\
\hline Hospitalization cost (USD) & $3408.9 \pm 592.6$ \\
\hline Overall complications & 3 \\
\hline Patients-related complications & $3(8.6)$ \\
\hline \multicolumn{2}{|l|}{ Intraoperative complications } \\
\hline Transfusion & 0 \\
\hline Injury & 0 \\
\hline \multicolumn{2}{|l|}{ Postoperative complications } \\
\hline Infection & $1(2.9)$ \\
\hline Urinary retention & $1(2.9)$ \\
\hline Persistent pain & 0 \\
\hline Haematoma & 0 \\
\hline Constipation & $1(2.9)$ \\
\hline Dyspareunia & 0 \\
\hline De novo SUI & $2(5.7)$ \\
\hline Deep vein thrombosis & 0 \\
\hline Follow-up duration (months) & $3.9 \pm 3.8(1-13)$ \\
\hline Recurrence & 0 \\
\hline
\end{tabular}

Values are shown as the mean \pm standard deviation (range) or $\mathrm{n}(\%)$

MUS surgery, and two underwent urethral folding. Twenty-four patients underwent anterior colporrhaphy, 24 patients underwent posterior colporrhaphy, 24 patients underwent perineal body repair, 2 patients underwent ovarian cystectomy, 10 patients underwent salpingo-oophorectomy, 2 patients underwent salpingectomy, and 1 patient underwent resection of a suspected malignant lesion on the surface of the colon. The mean operative duration (calculated from anaesthesia to the end of surgery, including other concurrent surgeries) was $111.7 \pm 39.4 \mathrm{~min}$. The mean blood loss was $67.9 \pm 35.8 \mathrm{ml}$. The mean length of postoperative hospital stay was $3.7 \pm 1.1$ days. The mean hospitalization cost was $3408.9 \pm 592.6$ dollars.

There were no cases of intraoperative blood transfusion or injury (Table 2). In total, 3 postoperative complications occurred in 3 patients (8.6\%). One patient (2.9\%) developed a urinary tract infection, tested positive for Escherichia coli, and was cured after antibacterial treatment. One patient (2.9\%) developed postoperative urinary retention; this patient underwent catheterization for urine drainage and was cured after 3 days of acupuncture treatment. One patient (2.9\%) suffered from constipation, which was relieved with a glycerine enema. There were no cases of de novo SUI, deep vein thrombosis, persistent pain, haematoma or dyspareunia as postoperative complications. The mean follow-up duration was $3.9 \pm 3.8$ (1-13) months. No patients had obvious recurrence.

Tables 3, 4 and 5 show the changes in the POP-Q, PFDI-20 and PISQ-12 scores, respectively, from preoperatively to postoperatively at the latest follow-up examination for each patient. All variables except the total vaginal length (TVL) in Table 3 showed significant improvement in physical prolapse after surgery. Seventeen patients (48.6\%) were followed for more than 3 months and completed the PFDI-20 (Table 4). The Pelvic Organ Prolapse Distress Inventory 6 (POPDI-6), Colorectal-Anal Distress Inventory 8 (CRADI-8), Urinary Distress Inventory Short

Table 3 Change in POP-Q score $(n=35)$

\begin{tabular}{llll}
\hline Variable & Preoperative & Postoperative & $\mathbf{P}$ \\
\hline $\mathrm{Aa}$ & $+0.6 \pm 1.7$ & $-2.9 \pm 0.2$ & $0.000^{*}$ \\
$\mathrm{Ba}$ & $+1.9 \pm 2.2$ & $-2.9 \pm 0.3$ & $0.000^{*}$ \\
$\mathrm{C}$ & $+1.5 \pm 2.2$ & $-6.9 \pm 0.9$ & $0.000^{*}$ \\
$\mathrm{Ap}$ & $-1.4 \pm 1.0$ & $-3.0 \pm 0.1$ & $0.000^{*}$ \\
$\mathrm{Bp}$ & $-1.1 \pm 1.4$ & $-2.9 \pm 0.1$ & $0.000^{*}$ \\
$\mathrm{TVL}$ & $+7.4 \pm 0.5$ & $+7.2 \pm 0.4$ & 0.058 \\
\hline
\end{tabular}

POP-Q Pelvic Organ Prolapse Quantification, $T V L$ total vaginal length

Values are shown as the mean \pm standard deviation. ${ }^{*} P<0.05$ was statistically significant

Table 4 Change in PFDI-20 score $(n=17)$

\begin{tabular}{lrll}
\hline Variable & Preoperative & Postoperative & P \\
\hline Quality of life & & & \\
POPDI-6 & $9.9 \pm 3.5$ & $0.9 \pm 1.9$ & $0.000^{*}$ \\
CRADI-8 & $2.5 \pm 3.0$ & $0.7 \pm 2.1$ & $0.047^{*}$ \\
UDI-6 & $7.5 \pm 4.4$ & $1.6 \pm 2.8$ & $0.000^{*}$ \\
Total PFDI-20 & $19.9 \pm 6.7$ & $3.2 \pm 5.4$ & $0.000^{*}$
\end{tabular}

POPDI-6 Pelvic Organ Prolapse Distress Inventory 6, CRADI-8 Colorectal Anal Distress Inventory 8, UDI-6 Urinary Distress Inventory 6, PFDI-20 Pelvic Floor Distress Inventory 20

Values are shown as the mean \pm standard deviation. ${ }^{*} \mathrm{P}<0.05$ was statistically significant 
Table 5 Change in PISQ-12 score $(n=9)$

\begin{tabular}{llll}
\hline Variable & Preoperative & Postoperative & P \\
\hline PISQ-12 & $24.8 \pm 2.3$ & $38.3 \pm 4.1$ & $0.000^{*}$
\end{tabular}

PISQ-12 Pelvic Organ Prolapse/Urinary Incontinence Sexual Questionnaire Short Form

Values are shown as the mean \pm standard deviation. ${ }^{*} \mathrm{P}<0.05$ was statistically significant

Form (UDI-6) and total PFDI-20 scores were significantly decreased after surgery, indicating notable alleviation of pelvic, urinary and colorectal symptoms. Nine patients (25.7\%) had recovered their sexual life and completed the PISQ-12 (Table 5). The PISQ-12 score was significantly increased after surgery, indicating notable improvement in quality of sexual life.

\section{Discussion}

In the present study, vNOTES for USLS resulted in marked improvement in both anatomical prolapse and quality of life without cases of conversion or serious perior postoperative complications. These results suggest that vNOTES for USLS may be a feasible technique for treating severe POP.

USLS is a classic surgical method for the treatment of middle compartment POP. vNOTES has the advantages of two traditional surgical approaches, including good exposure, no abdominal wound, and more accurate suture placement after confirmation of ischial spine localization. Studies have shown that the intermediate portion of the USL at the level of the ischial spine is the strongest. Management of the anterior vaginal wall is very important. First, anterior colporrhaphy was performed when the anterior compartment reached stage II prolapse. Lee et al. reported that concomitant anterior colporrhaphy at the time of USLS seemed to reduce the recurrence of anterior vaginal wall prolapse [15]. Second, in hysterectomy cases, the nonabsorbable sutures were attached to the ipsilateral cardinal ligament stump and the pubocervical fascia (fascia of the anterior vaginal wall); and the anterior vaginal wall was pulled up to achieve suspension of the vaginal vault and reduce recurrence. Lavelle et al. reported that USLS performed vaginally or with an abdominal approach via laparoscopy resulted in a higher prolapse recurrence rate than sacrocolpopexy for stage III prolapse [16]. vNOTES for USLS in this study presented good short-term efficacy in terms of both anatomical prolapse and quality of life, without serious complications. However, a comparative study with a larger number of patients and a longer follow-up period should be conducted.

POP is often accompanied by SUI, which is easy to identify when patients have symptoms. In this study, five patients were diagnosed with SUI by preoperative symptoms and urodynamic examination results, and all of them underwent concomitant anti-incontinence surgery. To date, there have been no cases of de novo SUI during follow-up. This is consistent with other reports in the literature; when POP is combined with SUI, prolapse repair surgery combined with anti-incontinence surgery can reduce the risk of postoperative de novo SUI $[17,18]$. Occult SUI may be originally obscured by organ prolapse, and de novo SUI may appear after anatomical restoration, which is why studies with a longer follow-up period should be conducted. Postoperative urinary retention has been reported in $13-32 \%$ of patients who undergo POP repair [11, 19-21]. Houlihan et al. reported that the rate of postoperative urinary retention was $31 \%$ in vaginal USLS patients and 15\% in laparoscopic USLS patients [11]. Yune et al. conducted a study to identify risk factors for postoperative urinary retention after POP repair and showed that surgical approach, age, parity, preoperative postvoid residual urine, and concomitant transvaginal anterior/posterior repair may be related factors [22]. In this study, the rate of postoperative urinary retention was $2.9 \%$, which is inconsistent with that reported in the literature. More in-depth research is required to explore this difference.

The results of this study suggest that sexual activity was restored 3 months after surgery. However, only nine of the 17 patients recovered their sexual life. There were many factors affecting the sex lives, such as age, surgery, spouse, psychological factors, and et al. In contrast with women in Western countries, Chinese women experience a rapid decrease in sexual need with age, especially after the age of 50 [23].

At our hospital [24], vNOTES for USLS required a shorter postoperative hospital stay and lower hospitalization costs than sacrocolpopexy, which significantly reduced the national medical insurance cost of vNOTES for USLS. The procedure was less expensive because there were fewer complications and the mesh did not need to be purchased.

This study has several limitations. First, the follow-up duration was relatively short, and the sample size was limited. Another, this was an observational study with no control group and there lied in the inherent weaknesses of retrospective studies. However, to our knowledge, there are few articles to describe the surgical outcomes of vNOTES for USLS, which may provide some references.

\section{Conclusions}

vNOTES for USLS, with promising short-term efficacy and safety, may be a feasible technique for the treatment of severe prolapse. Hence, additional studies with a larger 
number of patients and a longer follow-up period should be conducted.

\begin{abstract}
Abbreviations
VNOTES: Transvaginal natural orifice transluminal endoscopic surgery; USLS: Uterosacral ligament suspension; POP-Q: Pelvic Organ Prolapse Quantification; PISQ-12: Pelvic Organ Prolapse/Urinary Incontinence Sexual Questionnaire Short Form; PFDI-20: Pelvic Floor Inventory-20; TVL: Total vaginal length; POP: Pelvic organ prolapse; SUI: Stress urinary incontinence; USL: Uterosacral ligament.
\end{abstract}

\section{Acknowledgements}

Not applicable.

\section{Authors' contributions}

All authors have read and approved the manuscript. ZYL: Patient recruitment, data collection and analysis, manuscript writing. YSC: Data collection and analysis, statistical analysis, and manuscript writing. XJW: Patient recruitment, data collection. JWL: Patient recruitment, data collection. KQH: Study design, patient recruitment, data collection. CDH: Study design, patient recruitment, responsible surgeon. All authors read and approved the final manuscript.

\section{Funding}

No funding was provided for this study.

\section{Availability of data and materials}

The datasets used and analyzed during the current study available from the corresponding author on reasonable request.

\section{Declarations}

Ethics approval and consent to participate

This study was approved by the Ethics Committee of the Obstetrics and Gynecology Hospital of Fudan University (no. 2019-32). All patients were given the written informed consent to the surgical procedure and to the use of individual data for research.

\section{Consent for publication}

All patients were given the written informed consent to the surgical procedure and to the use of individual data for research.

\section{Competing interests}

The authors declare that they have no competing interests.

Received: 3 November 2020 Accepted: 28 May 2021

Published online: 08 June 2021

\section{References}

1. Maher C, Feiner B, Baessler K, Christmann-Schmid C, Haya N, Brown J. Surgery for women with anterior compartment prolapse. Cochrane Database Syst Rev. 2016;11(11):CD004014. https://doi.org/10.1002/14651 858.CD004014.pub6.

2. Wu JM, Matthews CA, Conover MM, Pate V, Jonsson FM. Lifetime risk of stress urinary incontinence or pelvic organ prolapse surgery. Obstet Gynecol. 2014;123(6):1201-6. https://doi.org/10.1097/AOG.0000000000 000286.

3. Altman D, Väyrynen T, Engh ME, Axelsen S, Falconer C; Nordic Transvaginal Mesh Group. Anterior colporrhaphy versus transvaginal mesh for pelvicorgan prolapse. N Engl J Med. 2011;364(19):1826-36. doi: https://doi.org/ 10.1056/NEJMoa1009521. (Erratum in: N Engl J Med. 2013;368(4):394. PMID: 21561348).

4. Dyer O. Transvaginal mesh: FDA orders remaining products off US market. BMJ. 2019;365:1839. https://doi.org/10.1136/bmj.l1839.

5. Silva WA, Pauls RN, Segal JL, Rooney CM, Kleeman SD, Karram MM. Uterosacral ligament vault suspension: five-year outcomes. Obstet Gynecol.
2006;108(2):255-63. https://doi.org/10.1097/01.AOG.0000224610.83158. 23.

6. Barber MD, Visco AG, Weidner AC, Amundsen CL, Bump RC. Bilateral uterosacral ligament vaginal vault suspension with site-specific endopelvic fascia defect repair for treatment of pelvic organ prolapse. Am J Obstet Gynecol. 2000;183(6):1402-10. https://doi.org/10.1067/mob.2000.111298. (discussion1410-1).

7. Margulies RU, Rogers MA, Morgan DM. Outcomes of transvaginal uterosacral ligament suspension: systematic review and metaanalysis. Am J Obstet Gynecol. 2010;202(2):124-34. https://doi.org/10.1016/j.ajog.2009. 07.052.

8. Unger CA, Walters MD, Ridgeway B, Jelovsek JE, Barber MD, Paraiso MF. Incidence of adverse events after uterosacral colpopexy for uterovaginal and posthysterectomy vault prolapse. Am J Obstet Gynecol. 2015;212(5): 6031. doi: https://doi.org/10.1016/j.ajog.2014.11.034. (Epub 2014 Nov 27).

9. Panico G, Campagna G, Caramazza D, Amato N, Ercoli A, Scambia G, Cervigni M, Zaccoletti R. Laparoscopic high uterosacral ligament suspension: an alternative route for a traditional technique. Int Urogynecol J. 2018;29(8):1227-9. https://doi.org/10.1007/s00192-018-3588-4 (Epub 2018 Mar 2).

10. Jan H, Ghai V, Doumouchtsis SK. Mesh-free laparoscopic high uterosacral ligament suspension during total laparoscopic hysterectomy for uterine prolapse. J Minim Invasive Gynecol. 2018;25(6):952-3. https://doi.org/10. 1016/j.jmig.2017.12.008 (Epub 2017 Dec 20).

11. Houlihan S, Kim-Fine S, Birch C, Tang S, Brennand EA. Uterosacral vault suspension (USLS) at the time of hysterectomy: laparoscopic versus vaginal approach. Int Urogynecol J. 2019;30(4):611-21. https://doi.org/10. 1007/s00192-018-3801-5 (Epub 2018 Nov 5).

12. Lowenstein L, Baekelandt J, Paz Y, Lauterbach R, Matanes E. Transvaginal Natural Orifice Transluminal Endoscopic Hysterectomy and Apical Suspension of the Vaginal Cuff to the Uterosacral Ligament. J Minim Invasive Gynecol. 2019;26(6):1015. https://doi.org/10.1016/j.jmig.2019.04.007 (Epub 2019 Apr 10).

13. Liu J, Lin Q, Zhou X, Wu C, Guan Z, Guan X. Techniques for apical prolapse management in transvaginal natural orifice transluminal endoscopic surgery high uterosacral ligament suspension. J Minim Invasive Gynecol. 2020. https://doi.org/10.1016/j.jmig.2020.10.017 (Epub ahead of print).

14. Kowalski JT, Mehr A, Cohen E, Bradley CS. Systematic review of definitions for success in pelvic organ prolapse surgery. Int Urogynecol J. 2018;29(11):1697-704. https://doi.org/10.1007/s00192-018-3755-7 (Epub 2018 Aug 24).

15. Lee SY, Jeon MJ. Anterior repair versus no anterior repair for anterior vaginal wall prolapse resolved under simulated apical support at the time of uterosacral ligament suspension. Int Urogynecol J. 2020;31(10):2043-9. https://doi.org/10.1007/s00192-020-04229-0 (Epub 2020 Feb 11).

16. Lavelle ES, Giugale LE, Winger DG, Wang L, Carter-Brooks CM, Shepherd JP. Prolapse recurrence following sacrocolpopexy vs uterosacral ligament suspension: a comparison stratified by Pelvic Organ Prolapse Quantification stage. Am J Obstet Gynecol. 2018;218(1):116.e1-116.e5. https://doi. org/10.1016/j.ajog.2017.09.015 (Epub 2017 Sep 23).

17. van der Ploeg JM, van der Steen A, Zwolsman S, van der Vaart CH, Roovers J. Prolapse surgery with or without incontinence procedure: a systematic review and meta-analysis. BJOG. 2018;125(3):289-97. https:// doi.org/10.1111/1471-0528.14943 (Epub 2017 Nov 13).

18. Baessler K, Christmann-Schmid C, Maher C, Haya N, Crawford TJ, Brown J. Surgery for women with pelvic organ prolapse with or without stress urinary incontinence. Cochrane Database Syst Rev. 2018;8(8):CD013108. https://doi.org/10.1002/14651858.CD013108.

19. Cohn JA, Ni S, Kaufman MR, Graves AJ, Penson DF, Dmochowski RR, Reynolds WS. Urinary retention and catheter use among U.S. female Medicare beneficiaries: Prevalence and risk factors. Neurourol Urodyn. 2017;36(8):2101-8. https://doi.org/10.1002/nau.23248 (Epub 2017 Mar 7).

20. Hakvoort RA, Dijkgraaf MG, Burger MP, Emanuel MH, Roovers JP. Predicting short-term urinary retention after vaginal prolapse surgery. Neurourol Urodyn. 2009;28(3):225-8. https://doi.org/10.1002/nau.20636.

21. Turner LC, Kantartzis K, Shepherd JP. Predictors of postoperative acute urinary retention in women undergoing minimally invasive sacral colpopexy. Female Pelvic Med Reconstr Surg. 2015;21(1):39-42. https://doi. org/10.1097/SPV.0000000000000110. 
22. Yune JJ, Cheng JW, Wagner H, Kim J, Hardesty JS, Siddighi S. Postoperative urinary retention after pelvic organ prolapse repair: Vaginal versus robotic transabdominal approach. Neurourol Urodyn. 2018;37(5):1794800. https://doi.org/10.1002/nau.23526 (Epub 2018 Mar 23).

23. Zhang C, Cui L, Zhang L, Shi C, Zang H. Sexual activity and function assessment in middle-aged Chinese women using the female sexual function index. Menopause. 2017;24(6):669-76. https://doi.org/10.1097/ GME.0000000000000812 (PMID: 28072610)

24. Li J, Hu C, Wang X, Hua K, Chen Y. Transvaginal single-port laparoscopic pelvic reconstruction with $Y$-shaped mesh: experiences of 93 cases. Int
Urogynecol J. 2020. https://doi.org/10.1007/s00192-020-04418-x (Epub ahead of print).

\section{Publisher's Note}

Springer Nature remains neutral with regard to jurisdictional claims in published maps and institutional affiliations.
Ready to submit your research? Choose BMC and benefit from:

- fast, convenient online submission

- thorough peer review by experienced researchers in your field

- rapid publication on acceptance

- support for research data, including large and complex data types

- gold Open Access which fosters wider collaboration and increased citations

- maximum visibility for your research: over 100M website views per year

At BMC, research is always in progress.

Learn more biomedcentral.com/submissions 